\title{
A NOTE ON ED DEGREES OF GROUP-STABLE SUBVARIETIES IN POLAR REPRESENTATIONS
}

\author{
ARTHUR BIK AND JAN DRAISMA
}

\begin{abstract}
Авstract. In a recent paper, Drusvyatskiy, Lee, Ottaviani, and Thomas establish a "transfer principle" by means of which the Euclidean distance degree of an orthogonally-stable matrix variety can be computed from the Euclidean distance degree of its intersection with a linear subspace. We generalise this principle.
\end{abstract}

\section{INTRODUCTION}

Fix a closed algebraic subvariety $X$ of a finite-dimensional complex vector space $V$ equipped with a non-degenerate symmetric bilinear form $\langle-\mid-\rangle: V \times V \rightarrow \mathbb{C}$. Denote by $X^{\text {reg }}$ the smooth locus in $X$. Then for a sufficiently general data point $u \in V$ the number

$$
\#\left\{x \in X^{\mathrm{reg}} \mid u-x \perp T_{x} X\right\}
$$

of $E D$ critical points for $u$ on $X$ is finite. Suppose that $V=\mathbb{C} \otimes_{\mathbb{R}} V_{\mathbb{R}}$, the bilinear form is the complexification of a Euclidean inner product on $V_{\mathbb{R}}$ and $X$ is the Zariskiclosure of a real algebraic variety $X_{\mathbb{R}}$ that has real smooth points, then this number is, for $u \in V$ sufficiently general, positive and independent of $u$ and is called the Euclidean distance degree (ED degree for short) of $X$ in $V$. See [DHOST]. Here, the ED degree counts the number of critical points in the smooth locus of $X$ of the distance function $d_{u}: X \rightarrow \mathbb{C}$ sending $x \mapsto\langle u-x \mid u-x\rangle$.

The goal of this note is to show that the ED degree of a variety $X$ with a suitable group action can sometimes be computed from that of a simpler variety $X_{0}$ obtained by slicing $X$ with a linear subspace of $V$.

For the simplest example of this phenomenon, let $C \subseteq \mathbb{C}^{2}$ be the unit circle with equation $x^{2}+y^{2}=1$ where $\mathbb{C}^{2}$ is equipped with the standard form. The ED degree of $C$ equals 2 and this is easily seen as follows. First, $C$ is $\mathrm{O}_{2}$-stable where $\mathrm{O}_{2}$ is the orthogonal group preserving the bilinear form. For all $u \in \mathbb{C}^{2}$ and $g \in \mathrm{O}_{2}$, the map $g$ restricts to a bijection between ED critical points on $C$ for $u$ and for $g u$. In particular, the number of ED critical points on $C$ for a sufficiently general point $u \in \mathbb{C}^{2}$ equals that number for $g u$, for any choice of $g \in \mathrm{O}_{2}$. We may assume that $u$ is not isotropic. Therefore, by choosing $g$ suitably, we may assume that $u$ lies on the horizontal axis. And then, since $u \not \perp T_{p} \mathrm{O}_{2} p=T_{p} C$ for any point $p \in C$ not on the horizontal axis, the search for ED critical points is reduced to the search for such points on the intersection of $C$ with the horizontal axis. Clearly, both of the intersection points are critical.

Both authors were partially supported by the NWO Vici grant entitled Stabilisation in Algebra and Geometry. 
In the paper [DLOT], a generalisation of this example is studied. They consider the vector space $V=\mathbb{C}^{n \times t}$ equipped with the trace bilinear form and with the group $G=\mathrm{O}_{n} \times \mathrm{O}_{t}$ acting by left and right multiplication. The variety $X$ is chosen as the Zariski-closure of an $\left(\mathrm{O}_{n}(\mathbb{R}) \times \mathrm{O}_{t}(\mathbb{R})\right)$-stable real algebraic variety $X_{\mathbb{R}}$ in $\mathbb{R}^{n \times t}$. This ensures that $X$ is $G$-stable. The horizontal line is generalised to the $\min (n, t)$ dimensional space $V_{0}$ of diagonal matrices in $V$. They then prove that the ED degree of $X$ in $V$ equals the ED degree of $X_{0}:=X \cap V_{0}$ in $V_{0}$. In the paper, $X_{0}$ is defined in an a priori different manner, namely, as the Zariski-closure of the intersection of $X_{\mathbb{R}}$ with $V_{0}$. That this is the same thing as the intersection of $X$ with $V_{0}$ is the content of [DLOT, Theorem 3.6], which is an application of the fact that the quotient map under a reductive (in fact, here finite) group sends closed, group-stable sets to closed sets.

Note that, like the unit circle and the horizontal line from the first example, the variety $X$ and the subspace $V_{0}$ satisfy the following conditions:

(1) For $v_{0} \in V_{0}$ sufficiently general, the vectorspace $V$ is the orthogonal direct sum of $V_{0}$ and $T_{v_{0}} G v_{0}$.

(2) The set $G X_{0}$ is dense in $X$.

The tangent space $T_{v_{0}} G v_{0}$ is equal to $g v_{0}$ where $\mathfrak{g}$ is the Lie algebra of $G$, consists of all pairs $(a, b)$ of skew-symmetric $n \times n$ and $t \times t$ matrices and acts by $(a, b) \cdot v=a v-v b$ for all $v \in V$ and $(a, b) \in \mathfrak{g}$. From the fact that the bilinear form $\langle-\mid-\rangle$ is $G$-invariant, it follows that $\langle(a, b) v \mid w\rangle+\langle v \mid(a, b) w\rangle=0$ for all $v, w \in V$ and $(a, b) \in \mathfrak{g}$. So condition (1) is equivalent to the statement that if $v_{0} \in V_{0}$ is sufficiently general, then $w \in V$ satisfies $\operatorname{Tr}\left((a w) v_{0}^{T}\right)=\operatorname{Tr}\left((w b) v_{0}^{T}\right)=0$ for all skew-symmetric $a \in \mathbb{C}^{n \times n}, b \in \mathbb{C}^{t \times t}$ if and only if $w$ is a diagonal matrix. Using that symmetric matrices form the orthogonal complement, with respect to the trace form, of the space of skewsymmetric matrices, this is the content of [DLOT, Lemma 4.7]. Condition (2) follows from the fact that the Zariski-dense subset of $X$ of real $n \times t$ matrices admit a singular value decomposition.

We will generalize the result of [DLOT] by showing that conditions (1) and (2) are sufficient for establishing that the ED degree of $X$ in $V$ equals that of $X_{0}$ in $V_{0}$, and we will describe the orthogonal representations that have such a subspace $V_{0}$-these turn out to be the polar representations of the title.

The remainder of the paper is organized as follows. In Section 2 we state our main results. Section 3 showcases a concrete optimization problem amenable to our techniques: given a real symmetric matrix, find a closest symmetric matrix with prescribed eigenvalues. In Section 4 we discuss the relation between complex varieties to which our theorem applies, acted upon by complex reductive groups; and their real counterparts acted upon by compact Lie groups. Section 5 contains the proof of our main theorem, and Section 6 discusses one possible approach for conclusively testing whether an orthogonal representation is polar. Finally, in Section 7 we discuss some of the most important polar representations coming from the irreducible real polar representation found in [Da]. 


\section{Main Results}

Let $V$ be a finite-dimensional complex vector space equipped with a nondegenerate symmetric bilinear form $\langle-\mid-\rangle: V \times V \rightarrow \mathbb{C}$. Let $G$ a complex algebraic group and let $G \rightarrow O(V)$ be an orthogonal representation.

Main Theorem. Suppose that $V$ has a linear subspace $V_{0}$ such that, for sufficiently general $v_{0} \in V_{0}$, the space $V$ is the orthogonal direct sum of $V_{0}$ and the tangent space $T_{v_{0}} G v_{0}$ of $v_{0}$ to its G-orbit. Let $X$ be a G-stable closed subvariety of $V$. Set $X_{0}:=X \cap V_{0}$ and suppose that $G X_{0}$ is dense in $X$. Then the $E D$ degree of $X$ in $V$ equals the $E D$ degree of $X_{0}$ in $V_{0}$.

Remark 1. The condition that for sufficiently general $v_{0} \in V_{0}$ the space $V$ is the orthogonal direct sum of $V_{0}$ and $T_{v_{0}} G v_{0}$ implies that the restriction of the form $\langle-\mid-\rangle$ to $V_{0}$ is non-degenerate and that $V_{0}$ and $T_{v_{0}} G v_{0}$ are perpendicular for all $v_{0} \in V_{0}$.

Remark 2. When $T_{x} X \cap\left(T_{x} X\right)^{\perp}=\{0\}$ for some $x \in X^{\text {reg }}$, then the ED degree of $X$ in $V$ is positive by [DHOST, Theorem 4.1]. Whenever $X$ is the complexification of a real variety with smooth points, this condition is satisfied. Also note that this condition implies that $T_{x} X_{0} \cap\left(T_{x} X_{0}\right)^{\perp}=\{0\}$ for some $x \in X_{0}^{\text {reg }}$, so that the ED degree of $X_{0}$ in $V_{0}$ is positive as well.

The (proof of the) Main Theorem has the following real analogue.

Theorem 3. Let $V_{\mathbb{R}}$ be a finite-dimensional real vector space equipped with a positive definite inner product. Let $K$ be a Lie group and let $K \rightarrow \mathrm{O}\left(V_{\mathbb{R}}\right)$ be an orthogonal representation. Suppose that $V_{\mathbb{R}}$ has a linear subspace $V_{\mathbb{R}, 0}$ such that, for sufficiently general $v_{0} \in V_{\mathbb{R}, 0}$, the space $V_{\mathbb{R}}$ is the orthogonal direct sum of $V_{\mathbb{R}, 0}$ and $T_{v_{0}} K v_{0}$. Then every K-orbit intersects $V_{\mathbb{R}, 0}$. Let $X$ be a real K-stable closed subvariety of $V_{\mathbb{R}}$ and set $X_{0}:=X \cap V_{\mathbb{R}, 0}$. Then the number of real critical points of the distance function to a point is constant along orbits of $K$ and the set of real critical points on $X$ for a sufficiently general $v_{0} \in V_{\mathbb{R}, 0}$ is contained in $X_{0}$.

Remark 4. When we consider an arbitrary $v_{0} \in V_{\mathbb{R}, 0}$, the space

$$
N_{v_{0}}=\left\{v \in V_{\mathbb{R}} \mid v \perp T_{v_{0}} K v_{0}\right\}
$$

contains $V_{\mathbb{R}, 0}$, but may be bigger. So while it is still true that the critical points on $X$ for $v_{0}$ are orthogonal to $T_{v_{0}} K v_{0}$, this does not imply that they lie in $V_{\mathbb{R}, 0}$. However, in this case the stabilizer $K_{v_{0}}$ acts on $N_{v_{0}}$ and by [DK. Theorem 2.4] this representation again satisfies the conditions of Theorem 3 with the subspace $V_{\mathbb{R}, 0}$ of $N_{v_{0}}$ again playing the same role. In particular, the $K_{v_{0}}$-orbit of any critical point on $X$ for $v_{0}$ intersects $V_{\mathbb{R}, 0}$. This allows us to still restrict the search for critical points on $X$ for $v_{0}$ to $X_{0}$. Since $K_{v_{0}}$ preserves the distance to $v_{0}$, the same is true for closest points on $X$ to $v_{0}$.

Apart from proving the Main Theorem, we also classify all orthogonal representations $G \rightarrow \mathrm{O}(V)$ for which a subspace $V_{0}$ as in the Main Theorem exists. Theorem 7 below relates this problem, in the case of reductive $G$, to the classification by Dadok and Kac of so-called polar representations [DK, Da].

Definition 5. A complex orthogonal representation $V$ of a reductive algebraic group $G$ is called stable polar when there exists a vector $v \in V$ such that the orbit $G v$ is closed and maximal-dimensional among all orbits of $G$ and such that the 
codimension of the subspace $\left\{x \in V_{\mathbb{C}} \mid \mathfrak{g} x \subseteq \mathfrak{g} v\right\}$ equals the dimension of $G v$ where $\mathfrak{g}$ is the (complex) Lie algebra of $G$.

Definition 6. A real orthogonal representation $V_{\mathbb{R}}$ of a compact Lie group $K$ is called polar when there exists a vector $v \in V_{\mathbb{R}}$ such that the orbit $K v$ is maximaldimensional among all orbits of $K$ and such that $\kappa u$ is perpendicular to $(\kappa v)^{\perp}$ for all $u \in(\kappa v)^{\perp}$ where $\kappa$ is the (real) Lie algebra of $K$.

Theorem 7. Let $V$ be an orthogonal representation of a reductive group $G$. Then the following are equivalent:

(i) V satisfies the conditions of the Main Theorem;

(ii) $V$ is a stable polar representation; and

(iii) $V$ is the complexification of a polar representation of a maximal compact Lie group K contained in $G$.

Remark 8. In (ii), we ask for the representation $V$ to be stable, i.e. for there to exist a $v \in V$ whose orbit is closed and maximal-dimensional among all orbits. This is a notion coming from Geometric Invariant Theory and should not be confused with the notion of a subset $X$ of $V$ being $G$-stable, i.e. having $g X \subseteq X$ for all $g \in G$.

The only places in this paper where the word stable refers to the notion from GIT are in Definition 5 and Theorem 7

Remark 9. Analogously to the equivalence (i) $\Leftrightarrow($ ii) of Theorem 7 , the conditions on $V_{\mathbb{R}}$ in Theorem 3 are equivalent to $V_{\mathbb{R}}$ being a polar representation.

In the paper $\mid \mathrm{Da}]$, the irreducible real polar representations of compact Lie groups are completely classified, giving us a list of spaces on which our Main Theorem can be applied. We discuss some of these spaces in section 7 .

\section{INTERLUDE: THE CLOSEST SYMMETRIC MATRIX WITH PRESCRIBED EIGENVALUES}

Given a symmetric matrix $A \in \mathbb{R}^{n \times n}$ and given a sequance of real numbers $\lambda=\left(\lambda_{1} \leq \lambda_{2} \leq \ldots \leq \lambda_{n}\right)$, how does one find the symmetric matrx $B \in \mathbb{R}^{n \times n}$ with spectrum $\lambda$ that minimizes $d_{A}(B):=\sum_{i, j}\left(a_{i j}-b_{i j}\right)^{2}$ ?

To cast this as an instance of Theorem 3 , take for $V_{\mathbb{R}}$ the space of real symmetric matrices acted upon by the group $K=\mathrm{O}_{n}(\mathbb{R})$ of orthogonal $n \times n$-matrices via the action $\alpha:(g, A) \mapsto g A g^{T}$. The $K$-invariant inner product on $V_{\mathbb{R}}$ is given by

$$
\langle C \mid D\rangle=\operatorname{Tr} C^{T} D=\sum_{i, j} c_{i j} d_{i j} .
$$

We claim that the space $V_{\mathbb{R}, 0}$ of diagonal matrices has the properties of Theorem 3 . Indeed, if $D$ is any diagonal matrix with distinct eigenvalues, then differentiating $\alpha$ and using that the Lie algebra $\kappa$ of $K$ is the Lie algebra of skew-symmetric matrices, we find that

$$
T_{D} K D=\left\{B D-D B \mid B^{T}=-B\right\}
$$

is precisely the space of symmetric matrices with zeroes on the diagonal, i.e., the orthogonal complement of $V_{\mathbb{R}, 0}$.

Let $X$ be the real-algebraic variety in $V_{\mathbb{R}}$ consisting of matrices with the prescribed spectrum $\lambda$. Then Theorem 3 says that, if $A$ lies in $V_{0}$, so that $A=$ $\operatorname{diag}\left(\mu_{1}, \ldots, \mu_{n}\right)$, then the critical points of $d_{A}$ on $X$ are the same as the critical points of the restriction of $d_{A}$ to $X_{0}:=X \cap V_{0}$. If the $\lambda_{i}$ are distinct, then this intersection consists of $n$ ! diagonal matrices, one for each permutation of the $\lambda_{i}$. 
Accordingly, the ED degree of the complexification of $X$ (the subject of the Main Theorem) is then $n$ !. If the $\lambda_{i}$ are not distinct but come with multiplicities $n_{1}, \ldots, n_{k}$ adding up to $n$, then the ED degree is the multinomial coefficient $\frac{n !}{n_{1} ! \cdots n !}$. The group $S_{n}$ here is the Weyl group from Section 5. In Example 23 we will see a large class of examples where the ED degree equals the order of the Weyl group.

Still assuming that $A$ is diagonal, we get a diagonal matrix $B \in X_{0}$ closest to $A$ by arranging the $\lambda_{i}$ in the same order as the $\mu_{i}$. To see this, let $\pi \in S_{n}$ be a permutation. If $\mu_{i}<\mu_{j}$ and $\lambda_{\pi(i)}>\lambda_{\pi(j)}$ for some $i, j \in[n]$, then

$$
\left(\mu_{i}-\lambda_{\pi(i)}\right)^{2}+\left(\mu_{j}-\lambda_{\pi(j)}\right)^{2}-\left(\mu_{i}-\lambda_{\pi(j)}\right)^{2}-\left(\mu_{j}-\lambda_{\pi(i)}\right)^{2}=2\left(\mu_{j}-\mu_{i}\right)\left(\lambda_{\pi(i)}-\lambda_{\pi(j)}\right)>0
$$

and so $\pi$ cannot minimize $\sum_{i}\left(\mu_{i}-\lambda_{\pi(i)}\right)^{2}$.

Now when $A$ is not diagonal to begin with, we first compute $g \in \mathrm{O}_{n}(\mathbb{R})$ such that $A_{0}:=g A g^{T}$ is diagonal, find a diagonal matrix $B_{0}$ closest to $A_{0}$ as above, and then $B:=g^{-1} B_{0} g^{-T}$ is a solution to the original problem. In the same manner, one obtains all critical points of $d_{A}$ from those of $d_{A_{0}}$.

\section{Real compact Versus complex Reductive}

We will use the correspondence between compact Lie groups and reductive complex linear algebraic groups.

\section{Theorem 10.}

(i) Any reductive complex algebraic group $G$ contains a maximal compact Lie group. All such subgroups are conjugate and Zariski dense in $G$.

(ii) Any compact Lie group is maximal in a reductive complex algebraic group, which is unique up to isomorphism.

Proof. See for example [Pr, Subsection 8.7.2] and [OV], Section 5.2].

The following lemma is well known, but included for completeness.

Lemma 11. The real orthogonal group $\mathrm{O}_{n}(\mathbb{R})$ is a maximal compact subgroup of the complex orthogonal group $\mathrm{O}_{n}$.

Proof. Any compact subgroup of $\mathrm{O}_{n}$ leaves invariant some Hermitian positivedefinite form on $\mathbb{C}^{n}$. The only Hermitian positive definite forms that are $\mathrm{O}_{n}(\mathbb{R})$ invariant are multiples of the standard form. So any compact subgroup of $\mathrm{O}_{n}$ containing $\mathrm{O}_{n}(\mathbb{R})$ is contained in the unitary group $U(n)$. Since $\mathrm{O}_{n}(\mathbb{R})=\mathrm{O}_{n} \cap U(n)$, we see that $\mathrm{O}_{n}(\mathbb{R})$ is maximal.

Let $G$ be a reductive linear algebraic group and let $K$ be a maximal compact Lie group contained in $G$. Then the complexification of any real representation of $K$ naturally has the structure of a representation of $G$.

Proposition 12. A (complex) representation of $G$ is orthogonal if and only if it is the complexification of a (real) representation of $K$ that is orthogonal with respect to some positive definite inner product.

Proof. Let $V$ be an orthogonal real representation of $K$ and let $V_{\mathbb{C}}$ be its complexification. Extend the inner product $\langle-\mid-\rangle$ on $V$ to a non-degenerate symmetric bilinear form on $V_{\mathbb{C}}$. Then $\langle v \mid w\rangle=\langle g v \mid g w\rangle$ for all $v, w \in V_{\mathbb{C}}$ and $g \in K$. So since $K$ is Zariski dense in $G$, we see that $V_{\mathbb{C}}$ is an orthogonal representation of $G$.

Let $V$ be an orthogonal complex representation of $G$. Then the image of $K$ in $O(V)$ is contained in some maximal compact subgroup $H$ of $O(V)$. Let $W$ be a 
real subspace of $V$ with $W \otimes \mathbb{C}=V$ such that the bilinear form on $V$ restricts to a $\mathbb{R}$-valued positive definite inner product on $W$. Since all maximal compact subgroups of $O(V)$ are conjugate, we see that

$$
H=g O(W) g^{-1}
$$

for some $g \in O(V)$. Let $V_{\mathbb{R}}$ be the real vector space $g W$ with inner product $\langle v \mid w\rangle_{V_{\mathbb{R}}}=\left\langle g^{-1} v \mid g^{-1} w\right\rangle$ for all $v, w \in V_{\mathbb{R}}$. Then $V_{\mathbb{R}}$ is an orthogonal representation of $K$ whose complexification is isomorphic to $V=W \otimes_{\mathbb{R}} \mathbb{C}$ by the map $g^{-1}$.

Let $\mathfrak{g}$ be the (complex) Lie algebra of $G$ and let $\kappa$ be the (real) Lie algebra of $K$. The following theorem is a reformulation of Theorem 7 .

Theorem 13. Let $V_{\mathbb{R}}$ be an orthogonal representation of $K$ and let $V_{\mathbb{C}}$ be its complexification. Then the following are equivalent:

(i) there exists a (complex) subspace $V_{\mathbb{C}, 0}$ of $V_{\mathbb{C}}$ such that, for $v_{0} \in V_{\mathbb{C}, 0}$ sufficientely general, the space $V_{\mathbb{C}}$ is the orthogonal direct sum of $V_{\mathbb{C}, 0}$ and $\mathfrak{g} v_{0}$;

(ii) there exists a vector $v \in V_{\mathbb{C}}$ such that the orbit $G v$ is closed and maximaldimensional among all orbits of $G$ and such that the codimension of the subspace $\left\{x \in V_{\mathbb{C}} \mathfrak{g} x \subseteq \mathfrak{g} v\right\}$ equals the dimension of $G v$; and

(iii) there exists a vector $v \in V_{\mathbb{R}}$ such that the orbit $K v$ is maximal-dimensional among all orbits of $K$ and such that $\kappa u$ is perpendicular to $(\kappa v)^{\perp}$ for all $u \in(\kappa v)^{\perp}$.

Proof.

(ii) $\Rightarrow$ (i) Let $v \in V_{\mathbb{C}}$ be a vector as in (ii) and take

$$
V_{\mathbb{C}, 0}=\left\{x \in V_{\mathbb{C}} \mid \mathfrak{g} x \subseteq \mathfrak{g} v\right\} .
$$

Then for $v_{0} \in V_{\mathbb{C}, 0}$ sufficiently general, we have $\mathfrak{g} v_{0}=\mathfrak{g} v$. So it suffices to prove that $V_{\mathbb{C}}$ is the orthogonal direct sum of $V_{\mathbb{C}, 0}$ and $\mathfrak{g} v$. By [DK. Corollary 2.5], we know that $V_{\mathbb{C}}$ is the direct sum of $V_{\mathbb{C}, 0}$ (there donoted $c_{v}$ ) and $\mathfrak{g} v$. We have

$$
\left\langle V_{\mathbb{C}, 0} \mid \mathfrak{g} v\right\rangle=-\left\langle\mathfrak{g} V_{\mathbb{C}, 0} \mid v\right\rangle=-\langle\mathfrak{g} v \mid v\rangle=\{0\}
$$

and therefore the direct sum is orthogonal.

(i) $\Rightarrow$ (iii) Let $V_{\mathbb{C}, 0}$ be a subspace as in (i) and let $U$ be a dense open subset of $V_{\mathbb{C}, 0}$ such that $V_{\mathbb{C}}$ is the orthogonal direct sum of $V_{\mathbb{C}, 0}$ and $\mathfrak{g} w$ for all $w \in U$. Then $G U$ is a dense constructible subset of $V_{\mathbb{C}}$ and hence contains a dense open subset of $V_{\mathbb{C}}$. Note that the dimension of the orbit of any element of $G U$ equals the codimension of $V_{\mathbb{C}, 0}$. So since $G U$ is dense in $V_{\mathbb{C}}$, we see that these orbits must be maximal-dimensional among all orbits of $G$. Since $V_{\mathbb{R}}$ is dense in $V_{\mathbb{C}}$, the intersection of $V_{\mathbb{R}}$ with $G U$ contains a vector $v=g w$ with $g \in G$ and $w \in U$. Since $v \in G U$, we see that

$$
\operatorname{dim}_{\mathbb{R}}(K v)=\operatorname{dim}_{\mathbb{R}}(\kappa v)=\operatorname{dim}_{\mathbb{C}}(\mathfrak{g} v)=\operatorname{dim}_{\mathbb{C}}(G v)
$$

is maximal among the dimensions of all orbits of $K$. The space $V_{\mathbb{C}}$ is the orthogonal direct sum of $g V_{\mathbb{C}, 0}$ and $\mathfrak{g} v$. Therefore we have

$$
(\kappa v)^{\perp}=(\mathfrak{g} v)^{\perp} \cap V_{\mathbb{R}} \subseteq g V_{\mathbb{C}, 0}
$$

and hence for all $u \in(\kappa v)^{\perp}$, we have

$$
\left\langle\kappa u \mid(\kappa v)^{\perp}\right\rangle \subseteq\left\langle\mathfrak{g} u \mid g V_{\mathbb{C}, 0}\right\rangle=\left\langle g \mathfrak{g} g^{-1} u \mid g V_{\mathbb{C}, 0}\right\rangle=\left\langle\mathfrak{g}\left(g^{-1} u\right) \mid V_{\mathbb{C}, 0}\right\rangle=\{0\} .
$$


(iii) $\Rightarrow$ (ii) Let $v \in V_{\mathbb{R}}$ be a vector as in (iii). Since $\langle a v \mid a v\rangle=\langle v \mid v\rangle$ for all $a \in K$, we have $\langle b v \mid v\rangle+\langle v \mid b v\rangle=0$ for all $b \in \kappa$. So $\langle\kappa v \mid v\rangle=\{0\}$ and $v$ satisfies the condition of [DK. Theorem 1.1], because $\langle\mathfrak{g} v, v\rangle=\mathbb{C} \otimes\langle\kappa v \mid v\rangle=\{0\}$. Note that the Hermitian form $\langle-,-\rangle$ on $V_{\mathbb{C}}$ in that theorem is the extension of the inner product on $V_{\mathbb{R}}$ and that it is not equal to our bilinear form $\langle-\mid-\rangle$ on $V_{\mathbb{C}}$. By part (i) of Theorem 1.1, the orbit $G v$ is closed. Since $K$ is dense in $G$ and since the function $(u \mapsto \operatorname{dim}(G u))$ is lower semicontinuous, we see that $\operatorname{dim}(G v)=\operatorname{dim}(K v)$ is maximal. As stated in the introduction of [DK], the dimension of $\left\{x \in V_{\mathbb{C}} \mathfrak{g} x \subseteq \mathfrak{g} v\right\}$ is always at most the codimension of a maximal-dimensional orbit of $G$. Since

$$
\mathbb{C} \otimes(\kappa v)^{\perp} \subseteq \mathbb{C} \otimes\left\{u \in V_{\mathbb{R}} \mid \kappa u \subseteq \kappa v\right\} \subseteq\left\{x \in V_{\mathbb{C}} \mid \mathfrak{g} x \subseteq \mathfrak{g} v\right\},
$$

we must have equality.

Example 14. Let $G$ be the group $\mathrm{SL}_{2}(\mathbb{C})$ and let $V_{\mathbb{C}}$ be the irreducible 5-dimensional representation of $\mathrm{SL}_{2}(\mathbb{C})$. So $V_{\mathbb{C}}$ is the set of homogeneous polynomials in $x$ and $y$ of degree 4 and

$$
\begin{aligned}
\mathfrak{s l}_{2}(\mathbb{C}) & \mapsto \operatorname{End}_{\mathbb{C}}\left(V_{\mathbb{C}}\right) \\
\left(\begin{array}{cc}
a & b \\
c & -a
\end{array}\right) & \mapsto a\left(x \frac{\partial}{\partial x}-y \frac{\partial}{\partial y}\right)+b x \frac{\partial}{\partial y}+c y \frac{\partial}{\partial x}
\end{aligned}
$$

is the corresponding representation of $\mathfrak{s l}_{2}(\mathbb{C})$. Let the non-degenerate symmetric bilinear form $\langle-\mid-\rangle$ on $V_{\mathbb{C}}$ be given by the Gram matrix

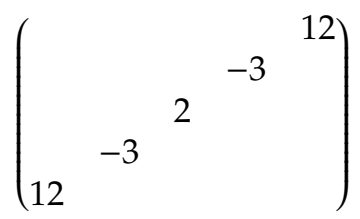

with respect to the basis $x^{4}, x y^{3}, x^{2} y^{2}, x y^{3}, y^{4}$ (obtained by setting $\left\langle x^{4} \mid y^{4}\right\rangle=12$ and using $\langle g v \mid w\rangle=-\langle x \mid g w\rangle$ for all $v, w \in V_{\mathbb{C}}$ and $g \in \mathfrak{s l}_{2}(\mathbb{C})$ ). Then $\langle-\mid-\rangle$ is $\mathrm{SL}_{2}(\mathbb{C})-$ invariant. A maximal compact subgroup of $\mathrm{SL}_{2}(\mathbb{C})$ is $K=\mathrm{SU}(2)$. The real subspace

$$
V_{\mathbb{R}}=\operatorname{span}_{\mathbb{R}}\left(x^{4}+y^{4}, i\left(x^{4}-y^{4}\right), x^{2} y^{2}, x y\left(x^{2}-y^{2}\right), i x y\left(x^{2}+y^{2}\right)\right) \text {. }
$$

of $V_{\mathbb{C}}$ is $\mathrm{SU}(2)$-stable and has $V_{\mathbb{C}}$ as its complexification. See the proofs of [IRS. Propositions 3 and 5] for how $V_{\mathbb{R}}$ was obtained. We will now check that the three equivalent conditions of the theorem are satisfied.

(i) Take $V_{\mathbb{C}, 0}=\operatorname{span}_{\mathbb{C}}\left(x^{4}+y^{4}, x^{2}+y^{2}\right)$. Then $V_{\mathbb{C}}$ is the orthogonal direct sum of $V_{\mathbb{C}, 0}$ and

$$
\mathfrak{s l}_{2}(\mathbb{C}) v_{0}=\operatorname{span}_{\mathbb{C}}\left(x^{4}-y^{4}, x^{3} y, x y^{3}\right)
$$

for all $v_{0}=a\left(x^{4}+y^{4}\right)+b x^{2} y^{2}$ with $4 a^{2} \neq b^{2}$.

(ii) Take $v=x^{4}+y^{4}+x^{2} y^{2}$. Then $\operatorname{dim}\left(\mathfrak{s I}_{2}(\mathbb{C}) v\right)=3=\operatorname{dim}\left(\mathrm{SL}_{2}(\mathbb{C})\right)$. Hence the dimension of $\mathrm{SL}_{2}(\mathbb{C}) v$ is maximal. As in the proof of the theorem, we see that the orbit $\mathrm{SL}_{2}(\mathbb{C}) v$ is closed and

$$
\left\{x \in V_{\mathbb{C}} \mid \mathfrak{s l}_{2}(\mathbb{C}) x \subseteq \mathfrak{s l}_{2}(\mathbb{C}) v\right\}=\operatorname{span}_{\mathbb{C}}\left(x^{4}+y^{4}, x^{2}+y^{2}\right)
$$

has dimension $5-3=2$. 
(iii) Again take $v=x^{4}+y^{4}+x^{2} y^{2}$. We have

$$
\mathfrak{s H}(2)=\operatorname{span}_{\mathbb{R}}\left(\left(\begin{array}{ll}
i & -i
\end{array}\right),\left(\begin{array}{ll} 
& -1 \\
1 &
\end{array}\right),\left(\begin{array}{ll} 
& i \\
i &
\end{array}\right)\right)
$$

and so we see that

$$
\mathfrak{s u}(2) v=\operatorname{span}_{\mathbb{R}}\left(i\left(x^{4}-y^{4}\right), x y\left(x^{2}-y^{2}\right), i x y\left(x^{2}+y^{2}\right)\right)
$$

has orthogonal complement

$$
\operatorname{span}_{\mathbb{R}}\left(x^{4}+y^{4}, x^{2} y^{2}\right)
$$

and we have $\mathfrak{H}(2) u \subseteq \mathfrak{H}(2) v$ for all $u$ in this complement.

\section{Proof of the Main Theorem}

Let $G \rightarrow O(V)$ be an orthogonal representation as in Section 2, Let $X$ be a $G$ stable closed subvariety of $V$. We assume the conditions of the Main Theorem. Note that if we replace $G$ by its unique irreducible component $G^{\circ}$ that contains the identity element, the conditions of the Main Theorem are still satisfied, because $G^{\circ}$ has finite index in $G$. So we may assume that $G$ is irreducible. This implies that all irreducible components of $X$ are also $G$-stable.

Lemma 15. The set $G V_{0}$ is dense in $V$.

Proof. The derivative of the multiplication map $G \times V_{0} \rightarrow V$ at a (smooth) point $\left(1, v_{0}\right)$ equals the map

$$
\begin{aligned}
\mathfrak{g} \oplus V_{0} & \rightarrow V \\
\left(A, u_{0}\right) & \mapsto A v_{0}+u_{0}
\end{aligned}
$$

and has image $\mathfrak{g} v_{0}+V_{0}$, which by assumption equals $V$ for sufficienly general $v_{0} \in V_{0}$. Hence the derivative is surjective at $\left(1, v_{0}\right)$ for some $v_{0} \in V_{0}$. Therefore the multiplication map is dominant and its image $G V_{0}$ is dense in $V$.

Lemma 16. For elements $g \in G$ and $u \in V$, the ED critical points for gu are obtained from those of $u$ by applying $g$.

Proof. Let $x$ be a point on $X$. The element $g \in G$ acts linearly and preserves $X$ and

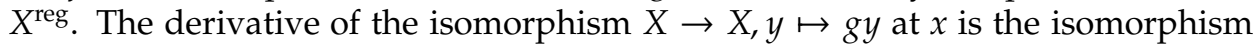
$T_{x} X \rightarrow T_{g x} X, w \mapsto g w$. So since $g$ also preserves the billinear form, we have $u-x \perp T_{x} X$ if and only if $g u-g x \perp T_{g x} X$.

Lemma 17. A sufficiently general $x_{0} \in X_{0}$ lies both in $X_{0}^{\text {reg }}$ and in $X^{\mathrm{reg}}$.

Proof. A sufficiently general point on $X_{0}$ lies in $X_{0}^{\text {reg }}$. Since $G X_{0}$ is constructible and dense in $X$, it contains a $G$-stable dense open subset $U$ of $X^{\text {reg }}$. The intersection of $U$ with $X_{0}$ is dense in $X_{0}$. Hence a sufficiently general point on $X_{0}$ lies in $X^{\text {reg }}$.

Define the Weyl group $W$ by

$$
W=N_{G}\left(V_{0}\right) / Z_{g}\left(V_{0}\right)=\left\{g \in G \mid g V_{0}=V_{0}\right\} /\left\{g \in G \mid g w=w \forall w \in V_{0}\right\} .
$$


Then the finite group $W$ acts naturally on $V_{0}$. Consider the set $S$ of $G$-stable closed subvarieties $Y$ of $V$ such that $G\left(Y \cap V_{0}\right)$ is dense in $Y$ and the set $R$ of $W$-stable closed subvarieties of $V_{0}$. Consider the maps

$$
\begin{aligned}
\varphi: \quad \rightarrow R & \rightarrow \text { and } \psi: R \\
Y & \mapsto Y \cap V_{0}
\end{aligned}
$$

between these two sets.

Lemma 18. The bijective maps $\varphi$ and $\psi$ are mutual inverses.

Proof. Since $S$ consists of the $G$-stable closed subvarieties $Y$ of $V$ such that $Y$ equals the closure of $G\left(Y \cap V_{0}\right)$ in $V$, we see that $\psi \circ \varphi$ is the identity map on $S$. Let $Z$ be a $W$ stable closed subvariety of $V_{0}$. It is clear that $Z \subseteq \varphi(\psi(Z))$ and we will show that in fact $\varphi(\psi(Z))=Z$ holds. Since $Z$ is $W$-stable and $W$ is finite, the variety $Z$ is defined by $W$-invariant polynomials $f_{1}, \ldots, f_{n} \in \mathbb{C}\left[V_{0}\right]^{W}$. By [DK, Theorem 2.9], there exists G-invariant polynomials $g_{1}, \ldots, g_{n} \in \mathbb{C}[V]^{G}$ such that $f_{i}$ is the restriction of $g_{i}$ to $V_{0}$ for all $i \in\{1, \ldots, n\}$. Since $g_{1}, \ldots, g_{n}$ are $G$-invariant and $g_{1}(z)=\cdots=g_{n}(z)=0$ for all $z \in Z$, we see that (the closure of) $G Z$ is contained in the zero set of the ideal generated by $g_{1}, \ldots, g_{n}$. Hence

$$
\varphi(\psi(Z))=\overline{G Z} \cap V_{0}
$$

is contained in the zero set of the ideal generated by the restrictions of $g_{1}, \ldots, g_{n}$ to $V_{0}$. This zero set is $Z$ and hence $\varphi(\psi(Z)) \subseteq Z$. So we see that $\varphi \circ \psi$ is the identity map on $R$.

Lemma 19. A sufficiently general $x_{0} \in X_{0}$ satisfies $T_{x_{0}} X=T_{x_{0}} X_{0}+T_{x_{0}} G x_{0}$.

Proof. By Lemma 17, we see that sufficiently general points of $X_{0}$ are contained in at most one irreducible component of $X$. Therefore each irreducible component of $X_{0}$ is contained in precisely one irreducible component of $X$. Let $Y$ be an irreducible component of $X$ and let $Z_{1}, \ldots, Z_{k}$ be the irreducible components of $X_{0}$ contained in $Y$. Then the Weyl group $W$ acts on the set $\left\{Z_{1}, \ldots, Z_{k}\right\}$. Since $G X_{0}$ is dense in $X$, we see that $G\left(Z_{1} \cup \cdots \cup Z_{k}\right)$ must be dense in $Y$. So $G Z_{i}$ must be dense in $Y$ for some $i \in\{1, \ldots, k\}$. By the previous lemma, for this $i$ we have

$$
Z_{1} \cup \cdots \cup Z_{k}=Y \cap V_{0}=\bigcup_{g \in W} g Z_{i}
$$

and hence $W$ must act transitively on $\left\{Z_{1}, \ldots, Z_{k}\right\}$. In particular, we see that $G Z_{j}$ is in fact dense in $Y$ for all $j \in\{1, \ldots, k\}$.

Take $Z=Z_{j}$ for any $j \in\{1, \ldots, k\}$. Then the multiplication map $G \times Z \rightarrow Y$ is dominant and $G$-equivariant when we let $G$ act on itself by left multiplication. Therefore its derivative at $(1, z)$ is surjective for $z \in Z$ sufficiently general. This means that $T_{z} Y=T_{z} Z+T_{z} G z$ for $z \in Z$ suffciently general. Since this holds for all components $Z$ of $X_{0}$, we see that $T_{x_{0}} X=T_{x_{0}} X_{0}+T_{x_{0}} G x_{0}$ for $x_{0} \in X_{0}$ suffciently general.

Lemma 20. Let $Y$ be a closed subvariety in a complex affine space $V$. Let $U$ be a dense open subset of $Y$ and let $Z$ be its complement in $Y$. Then for $v \in V$ sufficiently general, all ED critical points $y \in Y$ for $v$ lie in $U$.

Proof. See the proof of [DLOT, Lemma 4.2]. 
Lemma 21. Let $v_{0} \in V_{0}$ be sufficiently general. Then any ED critical point on $X_{0}$ for $v_{0}$ is an ED critical point on $X$ for $v_{0}$.

Proof. By combining the previous three lemmas, we may assume that all ED critical points $x_{0} \in X_{0}$ for $v_{0}$ are not only elements of $X_{0}^{\text {reg }}$ but also of $X^{\text {reg }}$ and that they satisfy $T_{x_{0}} X=T_{x_{0}} X_{0}+T_{x_{0}} G x_{0}$. Let $x_{0}$ be an ED critical points of $v_{0}$. Then $v_{0}-x_{0} \perp T_{x_{0}} X_{0}$ by criticality and $v_{0}-x_{0} \in V_{0} \perp T_{x_{0}} G x_{0}$ by the conditions of the Main Theorem (here we do not need that $T_{x_{0}} G x_{0}$ is the orthogonal complement of $V_{0}$-this may not be true-but only that it is contained in that complement). We see that

$$
v_{0}-x_{0} \perp T_{x_{0}} X_{0}+T_{x_{0}} G x_{0}=T_{x_{0}} X
$$

and hence $x_{0}$ is an ED critical point on $X$ for $v_{0}$.

Lemma 22. Let $v_{0} \in V_{0}$ be sufficiently general. Then any ED critical point on $X$ for $v_{0}$ is an ED critical point on $X_{0}$ for $v_{0}$.

Proof. Let $x \in X$ be an ED critical point for $v_{0}$. Then in particular $v_{0}-x \perp T_{x} G x=\mathfrak{g} x$. Together with $x \perp \mathfrak{g} x$, which holds by orthogonality of the representation, this implies that $v_{0} \perp \mathfrak{g} x$. Using once more the orthogonality of the representation, we see that $\left\langle x \mid \mathfrak{g} v_{0}\right\rangle=-\left\langle\mathfrak{g} x \mid v_{0}\right\rangle=\{0\}$. So $x \perp T_{v_{0}} G v_{0}$. Since $v_{0}$ is sufficiently general in $V_{0}$, the vector space $V$ is the orthogonal direct sum of $V_{0}$ and $T_{v_{0}} G v_{0}$ and therefore $x$ is an element of $V_{0}$. So since also $x \in X$, we have $x \in X_{0}$. Since $v_{0}-x \perp T_{x} X \supseteq T_{x} X_{0}$, we find that $x \in X_{0}$ is an ED critical point for $v_{0}$.

Proof of the Main Theorem. By Lemmas 15 and 16 we may assume that the sufficiently general point on $V$ is in fact a sufficiently general point $v_{0}$ on $V_{0}$. The previous two lemmas now tell us that the ED critical points for $v_{0}$ on $X$ and on $X_{0}$ are the same. Hence the ED degrees of $X$ in $V$ and $X_{0}$ in $V_{0}$ are equal.

Example 23. Let $G$ be a complex semisimple algebraic group acting on its Lie algebra $V=\mathfrak{g}$ by conjugation, let $V_{0}$ be a Cartan subalgebra of $\mathfrak{g}$ and let $W$ be the Weyl group. In Section 7, we show that $V$ satisfies the conditions of the Main Theorem. Suppose $X$ is the closed $G$-orbit of a sufficiently general point $v \in V_{0}$. Then the intersection $X_{0}=X \cap V_{0}$ is a single $W$-orbit by [DK. Theorem 2.8]. Since $X_{0}$ is the $W$-orbit of a sufficiently general point of $V_{0}$, it is a set of size \#W. So the ED degree of $X$ equals \#W.

Since $v$ is sufficienlty general, the codimension of $X$ in $V$ equals the dimension of $V_{0}$. So the degree of the variety $X$, i.e. the cardinality of $X \cap V^{\prime}$ for a sufficiently general subspace $V^{\prime}$ of $V$ with $\operatorname{dim}\left(V^{\prime}\right)=\operatorname{dim}\left(V_{0}\right)$, is at least the cardinality of $X \cap V_{0}$, which is the ED degree of $X$. Let $f_{1}, \ldots, f_{n}$ be a set of invariant polynomial generating the algebra $\mathbb{C}[V]^{G}$. Then, since $X$ is a closed G-orbit, we see that $X$ is defined by the equations $f_{1}=f_{1}(v), \ldots, f_{n}=f_{n}(v)$. Therefore the degree of $X$ is at most the product of the degrees of $f_{1}, \ldots, f_{n}$. By Theorem [Hu . Theorem 3.19], this product equals the size of the Weyl group $W$. So the degree and ED degree of $X$ are equal.

\section{Testing For the CONDitions of the Main TheOrem}

In the paper [Da], the irreducible polar representations of compact Lie groups are completely classified. Now suppose we have a not-necessarily irreducible orthogonal representation $V$ of a reductive algebraic group $G$. We would like to 
be able to test whether $V$ satisfies the conditions of the Main Theorem. In [DK. Section 2], some methods are given. In this section, we describe one more such method.

Lemma 24. For sufficiently general $v \in V$, the tangent space $\mathfrak{g} v$ of $v$ to its orbit is maximal-dimensional and non-degenerate with respect to the bilinear form.

Proof. By Proposition 12, we know that $V$ is the complexification of a real subspace $V_{\mathbb{R}}$ and that the $G$-invariant bilinear form $\langle-\mid-\rangle$ is the extension of a positive definite inner product on $V_{\mathbb{R}}$. Since $V_{\mathbb{R}}$ is dense in $V$ and the set of $v \in V$ such that the dimension of $\mathfrak{g} v$ is maximal is open and dense, there is an element $w$ in the intersection. Note that $\mathfrak{g} w=\kappa w \otimes_{\mathbb{R}} \mathbb{C}$. Since $\langle-\mid-\rangle$ is an inner product on $V_{\mathbb{R}}$, its restriction to $k w$ is non-degenerate. Therefore the restriction of $\langle-\mid-\rangle$ to $\mathfrak{g} w$ is non-degenerate as well.

Pick $\varphi_{1}, \ldots, \varphi_{n} \in \mathfrak{g}$ such that $\varphi_{1} w, \ldots, \varphi_{n} w$ form a basis of $\mathfrak{g} w$. Then the set of $v \in V$ such that $\varphi_{1} v, \ldots, \varphi_{n} v$ are linearly independent and the restriction of $\langle-\mid-\rangle$ to their span is non-degenerate is an non-empty open subset of $V$. Since the dimension of $\mathfrak{g} w$ is maximal, we see that for every element $v$ in this set, the tangent space $g v$ of $v$ to its orbit is spanned by $\varphi_{1} v, \ldots, \varphi_{n} v$. So for sufficiently general $v \in V$, the vector space $g v$ is non-degenerate with respect to the bilinear form.

Lemma 25. A subspace $V_{0}$ as in the Main Theorem exists if and only if for sufficiently general $v \in V$ and for all $u_{1}, u_{2} \perp \mathfrak{g} v$ we have $u_{1} \perp \mathfrak{g} u_{2}$.

Proof. Suppose such a subspace $V_{0}$ exists. Let $v \in V$ be sufficiently general and let $u_{1}, u_{2} \perp \mathfrak{g} v$. For $v_{0} \in V_{0}$ sufficiently general and for all $g \in G$, the vector space $V$ is the orthogonal direct sum of $g V_{0}$ and $g \mathfrak{g} v_{0}=\mathfrak{g}\left(g v_{0}\right)$. Since $G V_{0}$ contains an open dense subset of $V$, we may assume that $v=g v_{0}$ for such $v_{0}$ and $g$. So we see that $u_{1}, u_{2} \in g V_{0}$. We have $V_{0} \perp \mathfrak{g} u$ for all $u \in V_{0}$. Therefore we have $g V_{0} \perp \mathfrak{g} u$ for all $u \in g V_{0}$ and hence $u_{1} \perp \mathfrak{g} u_{2}$.

Let $v \in V$ be such that the $\mathfrak{g} v$ is maximal-dimensional, the restriction of the $G$-invariant bilinear form $\langle-\mid-\rangle$ to $\mathfrak{g} v$ is non-degenerate and $u_{1} \perp \mathfrak{g} u_{2}$ for all $u_{1}, u_{2} \perp \mathfrak{g} v$. Let $V_{0}$ be the orthogonal complement of $\mathfrak{g} v$. Then we see that $V_{0}$ is perpendicular to $\mathfrak{g} v_{0}$ for all $v_{0} \in V_{0}$. Let $v_{0} \in V_{0}$ be sufficiently general. Then we have $\mathfrak{g} v_{0}=\mathfrak{g} v$ and hence $V$ is the orthogonal direct sum of $V_{0}$ and $\mathfrak{g} v_{0}$.

Lemma 26. Let $W$ be a finite-dimensional complex vector space, let

$$
f_{1}, \ldots, f_{m}: V \rightarrow W
$$

be linear maps and let $w \in W$ be an element. Then the following are equivalent:

(i) For $v \in V$ sufficiently general, we have $w \in \operatorname{span}_{\mathbb{C}}\left(f_{1}(v), \ldots, f_{m}(v)\right)$.

(ii) We have $1 \otimes w \in \operatorname{span}_{\mathbb{C}\left(V^{*}\right)}\left(f_{1}, \ldots, f_{m}\right) \subset \mathbb{C}\left(V^{*}\right) \otimes_{\mathbb{C}} W$.

Proof. Suppose that

$$
1 \otimes w=c_{1} f_{1}+\cdots+c_{m} f_{m}
$$

for some $c_{1}, \ldots, c_{m} \in \mathbb{C}\left(V^{*}\right)$. Then

$$
w=c_{1}(v) f_{1}(v)+\cdots+c_{m}(v) f_{m}(v)
$$

for the dense open subset of $V$ consisting of all $v$ where $c_{1}, \ldots, c_{m}$ can be evaluated.

For the converse, suppose that for $v \in V$ sufficiently general we know that $w$ is contained in the span of $f_{1}(v), \ldots, f_{m}(v)$. We may assume that $f_{1}(v), \ldots, f_{m}(v)$ are linearly independent for $v \in V$ sufficiently general. Let $v \in V$ be such that 
$f_{1}(v), \ldots, f_{m}(v)$ are linearly independent and $w$ is contained in their span. Choose $w_{1}, \ldots, w_{k} \in W$ such that $f_{1}(v), \ldots, f_{m}(v), w_{1}, \ldots, w_{k}$ form a basis of $W$. Now note that $f_{1}(v), \ldots, f_{m}(v), w_{1}, \ldots, w_{k}$ form a basis of $W$ for $v \in V$ sufficiently general. By choosing a basis, we may assume that $W=\mathbb{C}^{n+k}$. This gives us a morphism

$$
\begin{aligned}
\varphi: V & \rightarrow \mathbb{C}^{(m+k) \times(m+k)} \\
v & \mapsto\left(\begin{array}{lllllll}
f_{1}(v) & \ldots & f_{m}(v) & w_{1} & \ldots & w_{k}
\end{array}\right)
\end{aligned}
$$

such that $\varphi(v)$ is invertible for $v \in V$ sufficiently general. Consider the coefficients of $\varphi(v)$ as elements of the field $\mathbb{C}\left(V^{*}\right)$ of rational functions on $V$. Then the matrix $\varphi(v)$ is invertible and $c(v)=\varphi(v)^{-1} w$ is a vector with coefficients in $\mathbb{C}\left(V^{*}\right)$. We have

$$
w=\varphi(v) c(v)=c_{1}(v) f_{1}(v)+\cdots+c_{m}(v) f_{m}(v)+c_{n+1}(v) w_{1}+\cdots+c_{n+k}(v) w_{k}
$$

for $v \in V$ sufficiently. Since we also know that $f_{1}(v), \ldots, f_{m}(v), w_{1}, \ldots, w_{k}$ form a basis and that $w$ is contained in the span of $f_{1}(v), \ldots, f_{m}(v)$ for $v \in V$ sufficiently general, we see that $c_{n+1}, \ldots, c_{n+k}$ all must be equal to the zero function. Hence

$$
1 \otimes w=c_{1} f_{1}+\cdots+c_{m} f_{m}
$$

is contained in the span of $f_{1}, \ldots, f_{m}$ inside $\mathbb{C}\left(V^{*}\right) \otimes W$.

Now we combine the previous two lemmas to reduce checking the existence of $V_{0}$ to a linear algebra problem over $\mathbb{C}\left(V^{*}\right)$. Take $U=W=V$ and consider $U$ and $W$ as affine spaces. Let $\varphi_{1}, \ldots, \varphi_{n}$ form a basis of $\mathfrak{g}$. By Lemma 25, we know that the representation $V$ satisfies the conditions of the Main Theorem if and only if, for $v \in V$ sufficiently general, the variety in $U \times W$ given by the linear equations

$$
\left\langle u \mid \varphi_{i} v\right\rangle=\left\langle w \mid \varphi_{i} v\right\rangle=0, \quad i=1, \ldots, n
$$

is contained in the variety given by the equations $\left\langle u \mid \varphi_{j} w\right\rangle=0$ for $j=1, \ldots, n$. The latter holds if and only if the polynomials $\left\langle u \mid \varphi_{j} w\right\rangle$ are contained in the ideal $I$ of the coordinate ring $\mathbb{C}[U \times W]$ generated by $\left\langle u \mid \varphi_{i} v\right\rangle$ and $\left\langle w \mid \varphi_{i} v\right\rangle$ for $i=1, \ldots, n$. The polynomial $\left\langle u \mid \varphi_{j} w\right\rangle$ is homogeneous of degree 2 . So for a fixed $v \in V$, it is contained in $I$ if and only if

$$
\left\langle u \mid \varphi_{j} w\right\rangle \in \operatorname{span}_{\mathbb{C}}\left(\mathbb{C}[U \times W]_{(1)} \cdot\left\{\left\langle u \mid \varphi_{i} v\right\rangle,\left\langle w \mid \varphi_{i} v\right\rangle \mid i=1, \ldots, n\right\}\right) .
$$

So by Lemma 26, we see that $V$ satisfies the conditions of the Main Theorem if and only if

$$
\left\langle u \mid \varphi_{j} w\right\rangle \in \operatorname{span}_{\mathbb{C}\left(V^{*}\right)}\left(\mathbb{C}[U \times W]_{(1)} \cdot\left\{\left\langle u \mid \varphi_{i} v\right\rangle,\left\langle w \mid \varphi_{i} v\right\rangle \mid i=1, \ldots, n\right\}\right)
$$

for all $j \in\{1, \ldots, n\}$. The latter condition can be checked efficiently on a computer, requiring as input the bilinear form $\langle-\mid-\rangle$ and the images in $\operatorname{End}(V)$ of a basis of $\mathfrak{g}$.

\section{EXAMPLES}

In this section we highlight some of the families of polar representations coming from [Da]. We also point out how some of these families are related by means of slice representations as defined in [DK]. Our Main Theorem can be applied to each of these families, thus generalizing [DLOT, Theorem 4.11].

Remark 27. A representation $V$ of a group $G$ satisfies the conditions of the Main Theorem if and only if the direct sum of $V$ with the trivial representation does.

Remark 28. Let $V$ be the orthogonal direct sum of two representations $V_{1}$ and $V_{2}$ of $G$. Then if $V$ satisfies the conditions of the Main Theorem, so do $V_{1}$ and $V_{2}$. 
7.1. Adjoint representations. Let $G$ be a complex semisimple algebraic group acting on its Lie algebra $\mathfrak{g}$ by conjugation. This representation is orthogonal with respect to the Killing form $B$ on $\mathfrak{g}$ defined by

$$
B(v, w)=\operatorname{Tr}(\operatorname{ad} v \operatorname{ad} w)
$$

for $v, w \in \mathfrak{g}$. Since $G$ is semisimple, we know that $B$ is non-degenerate. Since $G$ acts by conjugation, the tangent space of a point $v \in \mathfrak{g}$ to its orbit equals $[\mathfrak{g}, v]$. We have

$$
B(w,[\mathfrak{g}, \mathfrak{v}])=-B([w, v], \mathfrak{g})
$$

for all $w, v \in \mathfrak{g}$. So $w \perp \mathfrak{g} v$ if and only if $[v, w]=0$. Let $h \in \mathfrak{g}$ and suppose $[h, v] \perp \mathfrak{g} v$. Then $h \in \operatorname{ker}(\operatorname{ad} v)^{2}=\operatorname{ker}(\operatorname{ad} v)$ and hence $[h, v]=0$. Hence $\mathfrak{g} v$ is non-degenerate. Let $V_{0}$ be a Cartan subalgebra of g. Let $v \in V_{0}$ be sufficiently general and let $u_{1}, u_{2} \perp \mathfrak{g} v$. Then $V_{0}=C_{\mathfrak{g}}\left(V_{0}\right)=C_{\mathfrak{g}}(v)$. So we have $u_{1}, u_{2} \in V_{0}$ and hence $u_{1} \perp \mathfrak{g} u_{2}$.

7.2. Standard representations of groups of type $B$ and $D$. Let $n$ be a positive integer and let $G$ be the orthogonal group $\mathrm{O}(n)$ acting on $\mathbb{C}^{n}$ with the standard form. Let $V_{0}$ be the subspace of $V$ spanned by the first basis vector $e_{1}$. For all $v \in V_{0}$ non-zero, we have $\mathfrak{g} v=\left\{A e_{1} \mid A \in \mathfrak{g l}_{n}\right.$ skew-symmetric $\}=\operatorname{span}\left(e_{2}, \ldots, e_{n}\right)=V_{0}^{\perp}$.

7.3. Representations of groups of type $B$ and $D$ of highest weight $2 \lambda_{1}$. Let $n$ be a positive integer and let $G$ be the orthogonal group $\mathrm{SO}(n)$ acting on the vector space $V$ of symmetric $n \times n$ matrices with trace zero by conjugation. The bilinear form given by

$$
\langle A \mid B\rangle=\operatorname{Tr}\left(A^{T} B\right)
$$

for $A, B \in V$ is non-degenerate and $\mathrm{SO}(n)$-invariant. Let $V_{0}$ be the subspace of $V$ consisting of all diagonal matrices. For all $D \in V_{0}$ with pairwise distinct entries on the diagonal, we have $\mathfrak{g} \cdot D=\left\{A D-D A \mid A \in \mathfrak{g l}_{n}\right.$ skew-symmetric $\}=V_{0}^{\perp}$.

7.4. Tensor products of two standard representations of groups of type $B$ and $D$. Let $n \leq m$ be positive integers and let $G$ be the group $\mathrm{O}(n) \times \mathrm{O}(m)$ acting on $n \times m$ matrices by left and right multiplication. The bilinear form given by

$$
\langle A \mid B\rangle=\operatorname{Tr}\left(A^{T} B\right)
$$

for $A, B \in \mathbb{C}^{n \times m}$ is non-degenerate and $G$-invariant. The subspace $V_{0}$ of $\mathbb{C}^{n \times m}$ consisting of diagonal matrices satisfies the conditions of the Main Theorem.

Remark 29. Consider the matrix $v=\left(I_{n} 0\right) \in \mathbb{C}^{n \times m}$. The stabilizer of $v$ equals

$$
G_{v}=\left\{\left(g,\left(\begin{array}{ll}
g & 0 \\
0 & h
\end{array}\right)\right) \mid g \in \mathrm{O}(n), h \in \mathrm{O}(m-n)\right\}
$$

and the orthogonal complement of $\mathfrak{g} v$ equals the set of matrices of the form $(A 0)$ where $A$ is a symmetric $n \times n$ matrix. Ignoring the trivial action from $\mathrm{O}(m-n)$, we see that the slice representation of the element $v$ is the direct sum of the representation from the previous subsection and the trivial representation. 
7.5. Second alternating powers of standard representations of groups of type $C$. Let $n$ be a positive integer and let $G$ be the symplectic group

$$
\operatorname{Sp}(n)=\left\{A \in \mathrm{GL}_{2 n} \mid A\left(\begin{array}{cc}
0 & I_{n} \\
-I_{n} & 0
\end{array}\right) A^{T}=\left(\begin{array}{cc}
0 & I_{n} \\
-I_{n} & 0
\end{array}\right)\right\}
$$

acting on the second alternating power $\Lambda^{2} \mathbb{C}^{2 n}$ of the standard representation. The Lie algebra of $\operatorname{Sp}(n)$ equals

$$
\begin{aligned}
\mathfrak{s p}(n) & =\left\{A \in \mathfrak{g l}_{2 n} \mid A\left(\begin{array}{cc}
0 & I_{n} \\
-I_{n} & 0
\end{array}\right)+\left(\begin{array}{cc}
0 & I_{n} \\
-I_{n} & 0
\end{array}\right) A^{T}\right\} \\
& =\left\{\left(\begin{array}{ll}
X & Y \\
Z & \Theta
\end{array}\right) \in \mathfrak{g l}_{2 n} \mid \begin{array}{c}
Y=Y^{T}, Z=Z^{T} \\
X+\Theta^{T}=0
\end{array}\right\} .
\end{aligned}
$$

The $\operatorname{Sp}(n)$-invariant skew-symmetric form on $\mathbb{C}^{2 n}$ induces the bilinear form on $\Lambda^{2} \mathbb{C}^{2 n}$ given by

$$
\langle v \wedge w \mid x \wedge y\rangle=v^{T}\left(\begin{array}{cc}
0 & I_{n} \\
-I_{n} & 0
\end{array}\right) x \cdot w^{T}\left(\begin{array}{cc}
0 & I_{n} \\
-I_{n} & 0
\end{array}\right) y-v^{T}\left(\begin{array}{cc}
0 & I_{n} \\
-I_{n} & 0
\end{array}\right) y \cdot w^{T}\left(\begin{array}{cc}
0 & I_{n} \\
-I_{n} & 0
\end{array}\right) x
$$

for $v, w, x, y \in \mathbb{C}^{2 n}$. This form is symmetric, non-degenerate and $\operatorname{Sp}(2 n)$-invariant. Let $V_{0}$ be the subspace of $\Lambda^{2} \mathbb{C}^{2 n}$ spanned by $e_{i} \wedge e_{n+i}$ for $i=1, \ldots, n$. Then for any linear combination $v$ of $e_{1} \wedge e_{n+1}, \ldots, e_{n} \wedge e_{2 n}$ with only non-zero coefficients, the vector space $\Lambda^{2} \mathbb{C}^{2 n}$ is the orthogonal direct sum of $V_{0}$ and $g v$.

Remark 30. The paper [Da] tells us that $\Lambda^{2} \mathbb{C}^{2 n}$ is isomorphic to $\mathfrak{g l}_{2 n} / \mathfrak{s p}(n)$ acted on by $\operatorname{Sp}(n)$ by conjugation. In this case, the subspace $V_{0}$ of consists of matrices of the form

$$
\left(\begin{array}{ll}
D & 0 \\
0 & D
\end{array}\right)
$$

with $D \in \mathfrak{g l}_{n}$ diagonal.

7.6. Tensor products of two standard representations of groups of type $C$. Let $n \leq m$ be positive integers and let $G$ be the group $\operatorname{Sp}(n) \times \operatorname{Sp}(m)$ acting on $2 n \times 2 m$ matrices by left and right multiplication. The bilinear form is defined by

$$
\langle A \mid B\rangle=\operatorname{Tr}\left(\left(\begin{array}{cc}
0 & I_{n} \\
-I_{n} & 0
\end{array}\right) A\left(\begin{array}{cc}
0 & I_{m} \\
-I_{m} & 0
\end{array}\right) B^{T}\right)
$$

for all $A, B \in \mathbb{C}^{2 n \times 2 m}$. This form is symmetric, non-degenerate and $G$-invariant. Let $V_{0}$ be the subspace of $V$ consisting of matrices of the form

$$
\left(\begin{array}{cccc}
D & 0 & 0 & 0 \\
0 & 0 & D & 0
\end{array}\right)
$$

where $D$ is a diagonal $n \times n$ matrix. Then for every invertible diagonal $n \times n$ matrix $D$ whose squares of diagonal entries are pairwise distinct, the vector space $\mathbb{C}^{2 n \times 2 m}$ is the orthogonal direct sum of $V_{0}$ and

$$
\mathfrak{g} \cdot\left(\begin{array}{cccc}
D & 0 & 0 & 0 \\
0 & 0 & D & 0
\end{array}\right)=\mathfrak{s p}(n)\left(\begin{array}{cccc}
D & 0 & 0 & 0 \\
0 & 0 & D & 0
\end{array}\right)+\left(\begin{array}{cccc}
D & 0 & 0 & 0 \\
0 & 0 & D & 0
\end{array}\right) \mathfrak{s p}(m)
$$


Remark 31. The slice representation of

$$
\left(\begin{array}{cccc}
I_{n} & 0 & 0 & 0 \\
0 & 0 & I_{n} & 0
\end{array}\right)
$$

is a representation of $\operatorname{Sp}(n) \times \operatorname{Sp}(m-n)$ where the second factor acts trivially. Ignoring this factor, the slice representation is isomorphic to the representation $\mathfrak{g l}_{2 n} / \mathfrak{s p}(n)$ from the previous remark.

7.7. Direct sums of standard representations of groups of type $A$ and their duals. Let $n$ be a positive integer and let $G$ be the group $\mathrm{SL}_{n}$ acting on $\mathbb{C}^{n} \oplus \mathbb{C}^{n}$ by

$$
g \cdot(v, w)=\left(g v, g^{-T} w\right)
$$

for all $g \in \mathrm{SL}_{n}$ and $v, w \in \mathbb{C}^{n}$. Let the bilinear form be given by

$$
\langle(v, w) \mid(x, y)\rangle=v^{T} y+x^{T} w .
$$

for all $v, w, x, y \in \mathbb{C}^{n}$. This form is symmetric, non-degenerate and $\mathrm{SL}_{n}$-invariant. Let $V_{0}$ be the subspace of $\mathbb{C}^{n} \oplus \mathbb{C}^{n}$ spanned by $\left(e_{1}, e_{1}\right)$. Then $\mathbb{C}^{n} \oplus \mathbb{C}^{n}$ is the orthogonal direct sum of $V_{0}$ and $\mathfrak{g} \cdot v$ for all non-zero $v \in V_{0}$.

7.8. Direct sums of representations of groups of type $A$ of highest weight $2 \lambda_{1}$ and their duals. Let $n$ be a positive integer and let $G$ be the group $\mathrm{GL}_{n}$ acting on the vector space $V$ of pairs of symmetric $n \times n$ matrices by $g \cdot(A, B)=\left(g A g^{T}, g^{-T} B g^{-1}\right)$ for all $g \in \mathrm{GL}_{n}$ and $(A, B) \in V$. Let the bilinear form on $V$ be given by

$$
\langle(A, B) \mid(C, D)\rangle=\operatorname{Tr}(A D+B C)
$$

for all symmetric matrices $A, B, C, D \in \mathfrak{g l}_{n}$. Let $V_{0}$ be the subspace

$$
\left\{(D, D) \mid D \in \mathfrak{g l}_{n} \text { diagonal }\right\}
$$

of $V$. Then for every invertible diagonal $n \times n$ matrix $D$ whose squares of diagonal entries are pairwise distinct, the vector space $V$ is the orthogonal direct sum of $V_{0}$ and

$$
\mathfrak{g} \cdot(D, D)=\left\{\left(A D+D A^{T},-A^{T} D-D A\right) \mid A \in \mathfrak{g l}_{n}\right\} .
$$

Remark 32. The slice representation of $\left(I_{n}, I_{n}\right)$ is isomorphic to the set of symmetric $n \times n$ matrices acted on by $\mathrm{O}_{n}$ with conjugation.

7.9. Direct sums of representations of groups of type $A$ of highest weight $\lambda_{2}$ and their duals. Let $n$ be a positive integer and let $G$ be the group $\mathrm{GL}_{n}$ acting on the vector space $V$ of pairs of skew-symmetric $n \times n$ matrices by $g \cdot(A, B)=$ $\left(g A g^{T}, g^{-T} B g^{-1}\right)$ for all $g \in \mathrm{GL}_{n}$ and $(A, B) \in V$. Let the bilinear form on $V$ be given by

$$
\langle(A, B) \mid(C, D)\rangle=\operatorname{Tr}(A D+B C)
$$

for all skew-symmetric matrices $A, B, C, D \in \mathfrak{g l}_{n}$. Let $V_{0}$ be the subspace

$$
\left\{\left(\left(\begin{array}{cc}
0 & E \\
-E & 0
\end{array}\right),\left(\begin{array}{cc}
0 & E \\
-E & 0
\end{array}\right)\right) \mid E \in \mathfrak{g l}_{n / 2} \text { diagonal }\right\}
$$

of $V$ if $n$ is even and the subspace

$$
\left\{\left(\left(\begin{array}{ccc}
0 & 0 & E \\
0 & 0 & 0 \\
-E & 0 & 0
\end{array}\right),\left(\begin{array}{ccc}
0 & 0 & E \\
0 & 0 & 0 \\
-E & 0 & 0
\end{array}\right)\right) \mid E \in \mathrm{gl}_{(n-1) / 2} \text { diagonal }\right\}
$$


of $V$ if $n$ is odd. Then for every invertible diagonal $\lfloor n / 2\rfloor \times\lfloor n / 2\rfloor$ matrix $E$ whose squares of diagonal entries are pairwise distinct, the vector space $V$ is the orthogonal direct sum of $V_{0}$ and the tangent space at the corresponding element of $V_{0}$ to its orbit.

Remark 33. Suppose $n$ is even. Then the slice representation of

$$
\left(\begin{array}{cc}
0 & I_{n / 2} \\
-I_{n / 2} & 0
\end{array}\right)
$$

is isomorphic to the representation $\Lambda^{2} \mathbb{C}^{n}$ of $\operatorname{Sp}(n / 2)$.

7.10. Direct sums of standard representations of groups of type $C$ and their duals. Let $n$ be a positive integer and let $G$ be the group $\operatorname{Sp}(n)$ acting on the vectorspace $\mathbb{C}^{2 n} \oplus \mathbb{C}^{2 n}$ with the form given by

$$
\langle(v, w) \mid(x, y)\rangle=v^{T}\left(\begin{array}{cc}
o & I_{n} \\
-I_{n} & 0
\end{array}\right) y+x^{T}\left(\begin{array}{cc}
o & I_{n} \\
-I_{n} & 0
\end{array}\right) w
$$

for all $v, w, x, y \in \mathbb{C}^{2 n}$. Let $V_{0}$ be the subspace of $\mathbb{C}^{2 n} \oplus \mathbb{C}^{2 n}$ spanned by some $(v, w)$ with $v_{i}, w_{i} \neq 0$ for all $i$ and $v_{i} w_{j}-v_{j} w_{i} \neq 0$ for all $i \neq j$. Then it follows from the following lemma that $\mathbb{C}^{2 n} \oplus \mathbb{C}^{2 n}$ is the orthogonal direct sum of $V_{0}$ and the tangent space at any non-zero element $V_{0}$ of its orbit.

Lemma 34. Let $v, w, x, y \in \mathbb{C}^{m}$ be such that $v_{i}, w_{i} \neq 0$ for all $i$ and $v_{i} w_{j} \neq v_{j} w_{i}$ for all $i \neq j$. Then $v^{T} S y=x^{T} S w$ for all symmetric $m \times m$ matrices $S$ if and only if $(x, y)=\lambda(v, w)$ for some $\lambda \in \mathbb{C}$.

7.11. Tensor products of two direct sums of standard representations of groups of type $A$ and their duals. Let $n \leq m$ be positive integers and let $\mathrm{GL}_{n} \times \mathrm{GL}_{m}$ act on $\mathbb{C}^{n \times m} \oplus \mathbb{C}^{n \times m}$ by

$$
(g, h)(A, B)=\left(g A h^{T}, g^{-T} B h^{-1}\right)
$$

for all $g \in \mathrm{GL}_{n}, h \in \mathrm{GL}_{m}$ and $A, B \in \mathbb{C}^{n \times m}$. Let the bilinear form be given by

$$
\langle(A, B) \mid(C, D)\rangle=\operatorname{Tr}\left(A^{T} D+C^{T} B\right)
$$

for all $A, B, C, D \in \mathbb{C}^{n \times m}$. Let $V_{0}$ be the subspace

$$
\left\{((D 0),(D 0)) \mid D \in \mathfrak{g l}_{n} \text { diagonal }\right\}
$$

of $V$. Then for all invertible diagonal $n \times n$ matrices $D$ whose squares of diagonal entries are pairwise distinct, the vector space $\mathbb{C}^{n \times m} \oplus \mathbb{C}^{n \times m}$ is the orthogonal direct sum of $V_{0}$ and

$$
\mathfrak{g}((D 0),(D 0))=\left\{\left((A D 0)+(D 0) B^{T},\left(-A^{T} D 0\right)-(D 0) B\right) \mid A \in \mathfrak{g l}_{n}, B \in \mathfrak{g l}_{m}\right\} .
$$

Remark 35. The slice representation of the pair $\left(\left(I_{n} 0\right),\left(I_{n} 0\right)\right)$ is a representation of $\mathrm{GL}_{n} \times \mathrm{GL}_{m}$ where the second factor acts trivially. Ignoring this factor, we get the adjoint representation of $\mathrm{GL}_{n}$. 


\section{REFERENCES}

[DK] Jiri Dadok, Victor Kac, Polar representations, J. Algebra 92 (1985), no. 2, pp. 504-524.

[Da] Jiri Dadok, Polar coordinates induced by actions of compact Lie groups, Trans. Amer. Math. Soc. 288 (1985), no. 1, pp. 125-137.

[DHOST] Jan Draisma, Emil Horobet, Giorgio Ottaviani, Bernd Sturmfels, Rekha R. Thomas, The Euclidean distance degree of an algebraic variety, Found. Comput. Math. 16 (2016), pp. 99-149.

[DLOT] Dmitriy Drusvyatskiy, Hon-Leung Lee, Giorgio Ottaviani, Rekha R. Thomas, The Euclidean distance degree of orthogonally invariant matrix varieties, Israel J. Math. (2017).

[Hu] James E. Humphreys, Reflection Groups and Coxeter Groups, Cambridge University Press (1990).

[IRS] Gerald Itzkowitz, Sheldon Rothman, Helen Strassberg, A note on the real representations of $\mathrm{SU}(2, \mathbb{C})$, Journal of Pure and Applied Algebra 69 (1991), no. 3, pp. 285-294.

[OV] Arkadij L. Onishchik, Ernest B. Vinberg, Lie Groups and Algebraic Groups, Springer Science \& Business Media (2012)

[Pr] Claudio Procesi, Lie groups: an approach through invariants and representations, Springer New York (2007).

Universität Bern, Mathematisches Institut, Alpeneggstrasse 22, 3012 Bern

E-mail address: arthur.bik@math.unibe.ch

Universität Bern, Mathematisches Institut, Sidlerstrasse 5, 3012 Bern, and Eindhoven UniverSITY OF TECHNOLOGY

E-mail address: jan.draisma@math.unibe.ch 